\title{
Formación en Ética en las Organizaciones: Revisión de la Literatura
}

\author{
Juliana Toro(1), María del P. Rodríguez ${ }^{(2)}$ \\ (1) Universidad Nacional de Colombia Sede Manizales, Campus La Nubia, Grupo de Investigación Ethos - \\ Bloque Q, Manizales-Colombia (e-mail: jtoroa@unal.edu.co) \\ (2) Universidad Nacional de Colombia Sede Manizales, Campus La Nubia, Facultad de Ingeniería y \\ Arquitectura, Dpto. de Ingeniería Industrial, Manizales-Colombia. (e-mail: mdrodriguezco@unal.edu.co)
}

Recibido Jul. 27, 2016; Aceptado Sep. 30, 2016; Versión final Dic. 20, 2016, Publicado Abr. 2017

\begin{abstract}
Resumen
Este artículo identifica las tendencias de investigación sobre la formación en ética en las organizaciones. La metodología combina la búsqueda sistemática con la narrativa. La sistemática se realiza a través de la plataforma web Tree of Science que permite clasificar artículos con base en redes de citación bibliográfica. Se resaltan cuatro tendencias de investigación: 1) aspectos pedagógicos para la formación en ética en las organizaciones; 2 ) incidencia del género en la formación en ética en las organizaciones; 3 ) relaciones entre la formación en ética en las organizaciones y la toma de decisiones éticas y 4) relaciones entre la formación en ética y la cultura organizacional ética. Se concluye que los aspectos pedagógicos son los que han recibido mayor atención por los investigadores, mientras que las relaciones entre la formación en ética y la cultura organizacional ética aún no han sido estudiadas con suficiencia, encontrándose aquí una línea de investigación prometedora.
\end{abstract}

\section{Ethics Training in Organizations: Literature Review}

\begin{abstract}
This paper identifies research trends about ethics training in organizations. The methodology combines the systematic search with the narrative. Systematic search is done through the web platform Tree of Science, which allows papers classification based on bibliographic citation networks. Four trends research are highlighted: 1) pedagogical aspects for ethics training in organizations; 2 ) impact of gender in ethics training in organizations; 3) relationship between ethics training in organizations and ethical decision-making; and 4) relationships between ethics training in organizations and ethical organizational cultures. In conclusion, the pedagogical aspects are those that have received most attention by researchers, while relationships between ethics training in organizations and ethical organizational cultures have not been enough studied, finding here a promising line of research.
\end{abstract}

Keywords: ethics; business ethics; ethics training; teaching ethics 


\section{INTRODUCCIÓN}

La ética en las organizaciones es un tema relativamente nuevo para las ciencias de la gestión, pues solo hasta finales de los años setenta y primeros de los ochenta se consolidó como especialidad. En 1981 se creó el Business and Profesional Ethics Journal, en 1982 el Journal of Business Ethics y en 1991 el Business Ethics Quaterly, revistas científicas reconocidas por su aporte al campo (Chan et al., 2016). En los Estados Unidos los estudios difundidos, en un primer momento, se ocupaban en mayor medida de una perspectiva legal. Empero, en Europa se asumía una tendencia hacia la confianza y la autorregulación de las organizaciones, lo que desencadenó la creación en 1987 de la European Business Ethics Network (EBEN), red académica que hasta la fecha conserva este enfoque.

Con una comunidad académica en desarrollo, la ética organizacional fue estableciendo líneas de investigación para fortalecer su corpus teórico. Desde inicio de los años 80 se identificó el vínculo entre ética organizacional y formación en ética como una fuente de ideas para mejorar la sensibilidad ética de los integrantes de la organización y fortalecer el ethos institucional (McWilliams y Nahavandi, 2006). Por ejemplo, en 1974, la Agencia Internacional de Acreditación de las Escuelas de Negocios - AACSB comenzó a exigir la inclusión de la ética en sus programas; en 1991, definió unos estándares para la formación en ética en las escuelas de negocios y en 2003 y 2013, ratificó la enseñanza de la ética organizacional como un elemento principal para emitir su acreditación (AACSB, 2013). En la actualidad las instituciones de educación superior tienen la responsabilidad de dar a conocer a sus estudiantes los problemas éticos con que se enfrentarán en el mundo laboral y deben asegurarse que la enseñanza de la ética organizacional sea una parte integral del currículo de cualquier profesión. Sin embargo, aún es común encontrar universidades y facultades que no fomentan el desarrollo moral de sus estudiantes (Hummel et al., 2016).

Lo anterior, unido a los conflictos que tienen las organizaciones con sus grupos de interés y los escándalos de corrupción financiera que han sacudido al mundo en las últimas décadas (Soltani, 2014; Mathews y Gandel, 2015), han generado que la ética tome relevancia y se encuentre en la agenda de organizaciones y universidades. Investigadores están desarrollando estudios no sólo sobre la pertinencia de la formación en ética para contrarrestar la corrupción y para desarrollar culturas organizacionales éticas, sino también sobre el diseño de una variedad de métodos que permitan una formación ética efectiva (McWilliams y Nahavandi, 2006; Felton y Sims, 2005). Debido a que este es un tema emergente, se requiere reconocer y analizar las tendencias de estudio, como un punto de partida para consolidar su investigación y contribuir a abordar los desafíos éticos que enfrentan las organizaciones (Arce y Gentile, 2015), asunto que aborda este artículo.

La ética es entendida como una rama de la filosofía que reflexiona sobre la moral. En este contexto la moral se define como el conjunto de normas sobre los comportamientos permitidos y prohibidos que comparte un grupo humano y que pretende regular el comportamiento social (Rodríguez et al., 2016). En otras palabras, la ética reflexiona sobre la finalidad de la conducta humana, de las instituciones sociales y de la convivencia en general (Guisán, 1990). Es un tipo de saber que busca conducir bajo un sentido racional las acciones de las personas, por lo que asume el estudio de sus comportamientos desde lo teórico y lo práctico. Como parte de la sociedad los seres humanos tienen la necesidad de desenvolverse en un entramado de relaciones que implican la ética como dimensión humana. Es por ello que las cuestiones éticas no sólo involucran asuntos individuales, sino que también evolucionan a nivel grupal, organizacional y contextual. Aunque de lo anterior, se concluye que la ética estudia la moral, en el presente artículo se usarán las dos palabras indistintamente, lo cual no implica mayor contradicción, pues ambas están relacionadas al ser la segunda el objeto de estudio de la primera (Rodríguez et al., 2006).

La ética organizacional articula la ética y las organizaciones como sistemas sociales en los cuales convergen personas y se comparten objetivos. Guillén (2006) la define como el análisis de lo que conviene hacer o no hacer para la contribución al propio bien y al de la organización, en busca de la mejora de la calidad humana y el desarrollo de las potencialidades de las personas que las conforman. Es una disciplina que reflexiona sobre los comportamientos humanos desde la perspectiva de lo que es correcto o incorrecto en la administración y que deben ser contrastados dentro de una sociedad con conflictos e intereses que ponen al límite a los seres humanos. Para Rodríguez et al. (2016) desarrollar competencias éticas es una necesidad para las organizaciones, pues la ética ayuda a sus integrantes a tomar mejores decisiones a partir de la reflexión sobre las normas que rigen sus comportamientos. Por ello, no es gratuito que la Teoría del Desarrollo Moral Cognitivo de Kohlberg (1976), sea una de las más empleadas para explicar los procesos mediante los cuales las personas van avanzando en la toma de decisiones éticas (Treviño, 1986; MacLagan, 2015), tal como se ahondará en uno de los apartados de este artículo.

Para efectos de esta revisión, se privilegia el uso de la palabra organización sobre términos como empresa, compañía, negocio o corporación, pues se considera que al hablar de organización se están incluyendo diversas instituciones sin importar su naturaleza (pública, privada, mixta, con o sin ánimo de lucro). 
Asimismo, el concepto de formación se asume desde una perspectiva más pragmática que reflexiva, donde la acepción tiene un carácter aplicado y de conocimiento específico que se dirige a la acción (De la Torre, 1997) teniendo como base el concepto aportado por Buckley y Caple en el que la formación "implica la adquisición de conductas, hechos, ideas, etc., que se definen fácilmente en un contexto de trabajo específico" (1991, p. 2). Es decir, se entiende la formación como un proceso que incluye contenidos que generan reflexión y fortalecen los valores del sujeto en un contexto dado y que está encaminado al desarrollo de saberes y destrezas para la vida personal, social, laboral o académica, de acuerdo con los intereses de la persona y, en este caso, de la organización. También se aclara que en la literatura sobre formación en ética en las organizaciones es usual encontrar términos que se usan indistintamente, por ejemplo: entrenamiento, enseñanza, aprendizaje y educación, por lo cual en la presente revisión se tienen en cuenta estas semejanzas, pero se conservan sus particularidades conceptuales.

Los estudios teóricos y empíricos sobre formación en ética han aumentado y las revistas académicas que publican sobre ética organizacional cada vez incluyen con mayor frecuencia el tema de formación. Se aprecia una evolución de la discusión hacia la efectividad de los programas de formación en ética (Verma et al., 2016) al superar el debate sobre la importancia de este tipo de formación. En este sentido, hay cierto acuerdo en la comunidad científica respecto a que la formación en ética puede sensibilizar a los empleados de una organización hacia la toma de decisiones y los comportamientos fundamentados en principios éticos (Rodríguez et al., 2016; Verma et al., 2016; Arce y Gentile, 2015; Weber, 2015, 1990; Warren et al., 2014; McWilliams y Nahavandi, 2006; Ritter, 2006; Adkins y Radtke, 2004).

Este artículo ilustra el panorama de la formación en ética en las organizaciones y asume una visión integradora que busca patrones comunes y puntos de divergencia en la literatura revisada (Velásquez, 2014). Para ello, se presentan cuatro secciones: la primera ilustra el método utilizado, el cual articula la búsqueda narrativa con la sistemática, esta última a través de la plataforma web Tree of Science (Robledo et al., 2014). La segunda establece tendencias de investigación que permiten abordar el asunto desde diversas perspectivas, entre las cuales se destacan las cuestiones metodológicas que buscan dar respuesta a cómo llevar a cabo este proceso formativo y la relación de la formación en ética con el género, la toma de decisiones y la cultura organizacional. La tercera discute los resultados y futuras líneas de investigación. Finalmente, la cuarta parte establece las principales conclusiones.

\section{MÉTODO}

Realizar una revisión sistemática de literatura implica recolectar, organizar, evaluar y sintetizar evidencias disponibles respecto a un tema de interés (Velásquez, 2014). Para construir la presente revisión, se realizó una sinergia entre la búsqueda narrativa con la búsqueda sistemática, haciendo énfasis en esta última (Ferreira et al., 2011). Como estrategia principal de la búsqueda sistemática se usó la herramienta web "Tree of Science - ToS", la cual utiliza la base de datos Thomson Reuters, Web of Science - WoS. El ToS aplica la teoría de grafos para encontrar los artículos más pertinentes a través del análisis de redes de citaciones, es decir, los artículos se clasifican de acuerdo con el volumen de citas que tienen en la comunidad científica (grado de entrada, intermediación y grado de salida). Esta clasificación ubica los artículos encontrados en "raíces" o lo que se podría denominar como clásicos, por ser los referentes del tema y por ende los más citados; "tronco" por ser fuentes bibliográficas con un alto grado de intermediación (citan artículos y son citados) y "hojas" en los cuales se encuentran artículos recientes que referencian autores de esa comunidad científica. La metáfora del árbol permite relacionar cada nodo con un artículo (Robledo et al., 2014).

La consulta en la WoS se hizo en febrero de 2016, con la ecuación de búsqueda: Tema=("business ethics") AND Título=("training" or "teaching" or "learning" or "education") Timespan=All years. Databases=SClEXPANDED, SSCI, A\&HCI. Dentro de la ecuación se estipuló que los conceptos podían ser referenciados en el título, en las palabras clave o en el resumen. La búsqueda en la WoS arrojó un total de 138 artículos, los cuales se exportaron a la plataforma ToS que de manera automática y a través de la aplicación de su algoritmo de búsqueda y selección generó un árbol con 80 artículos de diferentes bases de datos, clasificados en 10 raíces, 10 del tronco y 60 hojas (ver figura 1). El análisis preliminar de estos artículos conllevó a otras fuentes bibliográficas de interés, generando así una amplia red con información selectiva.

Las referencias obtenidas de la revisión sistemática, se complementaron con una revisión narrativa a través de una búsqueda manual en bases de datos. El objetivo era encontrar artículos publicados en los últimos 10 años en revistas académicas de corriente principal que publican sobre ética organizacional. Esta búsqueda cubrió el 7,84\% de las referencias encontradas, ya que algunos de los artículos consultados se encontraron a través de ambas revisiones (sistemática y narrativa), lo que permitió validar la herramienta web Tree Of Sience y encontrar patrones comunes en la literatura y en las fuentes de consulta. 

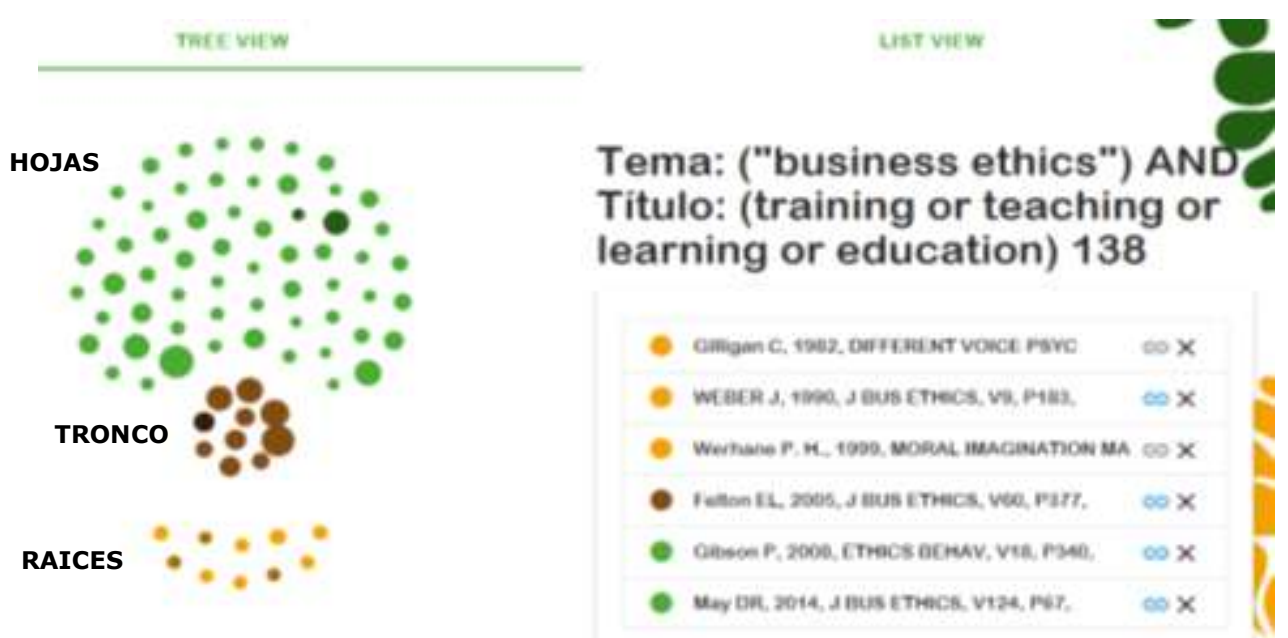

Fig.1: Árbol generado por Tree of Science - ToS

En 30 revistas diferentes se encontraron los 102 artículos revisados, sin embargo, las revistas que arrojaron mayor número de artículos con relación directa sobre el tema fueron: Journal of Business Ethics - JOBE (52), Academy of Management Learning \& Education - AMLE (7), Business Ethics Quaterly - BEQ (5), y Business Ethics: A European Review - BEER (4), las cuales son un referente de difusión científica sobre el campo de estudio (Chan et al., 2016). Se destaca que la mayoría de referencias seleccionadas fueron publicaciones del Journal of Business Ethics (50,98\% de los artículos consultados), este alto porcentaje se debe a que JOBE publica 28 ejemplares anuales, mientras otras de igual categoría como AMLE, BEQ y BEER publican 4, o por ejemplo Journal of Business Ethics Education que tiene 1 volumen anual o Teaching Business Ethics que estuvo activa solo desde 1997 hasta el año 2003. El volumen de publicaciones encontradas en medios relevantes indica que la producción académica en ética organizacional está identificando la formación en ética como un tema emergente.

\section{RESULTADOS Y DISCUSIÓN}

Después de tener los 80 artículos del ToS y el material bibliográfico adicional (22 referencias de la búsqueda narrativa) se procedió a su lectura completa. Para el análisis se hicieron diversas clasificaciones, aunque por cuestiones de espacio en el presente artículo se referencian las que más aportan a la discusión (según juicio de las autoras). Las categorizaciones se realizaron de manera inductiva. Finalmente se agruparon temáticas afines, logrando la identificación de tendencias de investigación.

Como punto de partida se hizo un registro de los artículos revisados por año (Tabla 1), lo que permitió identificar que en los 90 s ya se mencionaba el tema en la comunidad científica. Sin embargo, es a partir del año 2005 que se encuentra regularidad en la publicación de estudios. Vale la pena resaltar que 56 de los artículos revisados han sido publicados en los últimos 5 años, lo que evidencia la emergencia del tema.

Tabla 1: Año de publicación de los artículos consultados

\begin{tabular}{|c|c|c|c|c|c|c|c|c|c|c|c|c|c|}
\hline Año & 1969 & 1982 & 1986 & 1990 & 1991 & 1996 & 1998 & 1999 & 2000 & 2001 & 2002 & 2003 & 2004 \\
\hline$N^{\circ}$ Artículos & 1 & 1 & 2 & 1 & 1 & 1 & 1 & 2 & 1 & 1 & 3 & 1 & 1 \\
\hline Año & & 2005 & 2006 & 2007 & 2008 & 2009 & 2010 & 2011 & 2012 & 2013 & 2014 & 2015 & 2016 \\
\hline$N^{\circ}$ Artículos & & 4 & 7 & 3 & 4 & 6 & 1 & 4 & 8 & 7 & 15 & 22 & 4 \\
\hline
\end{tabular}

Para un análisis más detallado se hizo una categorización referente al proceso utilizado por cada investigador en su estudio, lo cual permitió identificar los enfoques de investigación prevalecientes. Como el campo disciplinar está enmarcado dentro de las ciencias fácticas y hay múltiples posturas epistemológicas respecto a la forma de hacer ciencia (Bunge, 1978), inicialmente se recurre a la clasificación genérica en cualitativa y cuantitativa por ser la más recurrente en la literatura respecto a investigación (Hernandez et al., 2010) (ver Tabla 2a). Estos resultados se complementan con una clasificación en otras tres posibilidades: teóricos, empíricos y empírico-analíticos, los cuales se asumen de acuerdo a lo planteado por Calderón (2005) quién afirma que una investigación puede ser teórica si su análisis se hace con el pensamiento y empírica cuando se confrontan datos y hechos. Por su parte las empírico-analíticas son entendidas como una sinergia entre ambos conceptos y en esta categoría se ubican los estudios que validan sus construcciones teóricas a través de su aplicación (ver Tabla 2b). 
Tabla 2a. Artículos con enfoques Cuantitativo/Cualitativo

\begin{tabular}{lrr}
\hline Tipo de estudio & No Artículos & Porcentaje \\
\hline Cuantitativos & 59 & $57,84 \%$ \\
\hline Cualitativos & 43 & $42,16 \%$ \\
\hline Total & 102 & $100 \%$ \\
\hline
\end{tabular}

Tabla 2b. Artículos con enfoques Teóricos/Empíricos

\begin{tabular}{lrr}
\hline Tipo de estudio & № Artículos & Porcentaje \\
\hline Empírico-Analíticos & 56 & $54,90 \%$ \\
\hline Empíricos & 25 & $24,51 \%$ \\
\hline Teóricos & 21 & $20,59 \%$ \\
\hline Total & 102 & $100 \%$ \\
\hline
\end{tabular}

En cuanto al enfoque, predominan las investigaciones cuantitativas, especialmente aquellas que contrastan modelos o metodologías de enseñanza de la ética en la práctica. Por su parte las cualitativas se destacan por hacer interpretaciones rigurosas de los supuestos que sustentan la formación en ética en las organizaciones. Estos resultados se relacionan con la segunda clasificación, donde llama la atención que las investigaciones que tienen un componente empírico son las más usuales $(79,41 \%)$, aunque cada cual se centra en sus propias variables, sin integrar técnicas o resultados previos. Esto indica que en las investigaciones sobre formación en ética organizacional se requiere la aplicación de métodos que permitan establecer patrones de medición comunes, de tal manera que se vayan consolidando posibles generalizaciones. También se requiere que las muestras de los estudios sean significativas y que se lleven a cabo estudios longitudinales que posibiliten la validación de los resultados a largo plazo (Jonson et al., 2015; Weber, 2015, 1990; Warren et al., 2014; Falkenberg y Woiceshyn, 2008; Adkins y Radtke, 2004).

La siguiente clasificación divide los artículos entre los que asumen la enseñanza de la ética como un proceso efectivo y los que cuestionan su efectividad (ver Tabla 3). Cuando se habla de efectividad, se hace referencia a los resultados positivos derivados de los aprendizajes que las personas obtuvieron durante el proceso formativo. Es decir, se considera que la formación en ética genera que un individuo amplíe su comprensión y raciocinio frente a los asuntos morales y mejore su capacidad para tomar decisiones basadas en principios éticos (Verma et al., 2016; Fischbach, 2015; Cameron y O'Leary, 2015; Baden, 2014; Weber, 2015, 1990; Hare, 1975).

Tabla 3: Artículos que plantean discusión sobre efectividad de la formación en ética

\begin{tabular}{lcc}
\hline Discusión sobre efectividad de la formación en ética & No Artículos & Porcentaje \\
\hline Artículos que plantean argumentos sobre la efectividad o no del proceso formativo & 62 & $60,78 \%$ \\
\hline Artículos que asumen la formación en ética como un proceso efectivo & 40 & $39,22 \%$ \\
\hline Total & 102 & $100 \%$ \\
\hline
\end{tabular}

En la tabla 3 se aprecia que el $60,78 \%$ de los artículos siguen discutiendo sobre el tema, cuestionando por ejemplo el aprendizaje sobre lo ético que puede obtener un adulto (Wang y Calvano, 2015; Gu y Neesham, 2014; Castaño y Garín, 2012; McWilliams y Nahavand, 2006; Sims y Felton, 2006). El 39,22\% da por hecho que la formación en ética organizacional es un proceso que genera buenos resultados, lo que le permite a estos autores enfocarse en cuestiones más específicas, por ejemplo validación de metodologías o propuestas pedagógicas para mejorar el proceso de enseñanza-aprendizaje en diferentes contextos.

Otra clasificación hace referencia a los contenidos de cada artículo. Se aclara que hay artículos que abordan dos o más categorías, pero se clasifican en una sola, según su temática predominante (ver Tabla 4). El primer tema se refiere a aspectos pedagógicos y didácticos que se deben tener en cuenta para enseñar ética en las organizaciones, se abordan asuntos como métodos de aprendizaje, recursos necesarios, ejes conceptuales, rol de los participantes, entre otros. En el segundo se encuentran artículos que discuten o aportan evidencia respecto al estado o la relevancia que se le da a la enseñanza de la ética organizacional en las universidades, mientras que en el tercero se ubican textos que profundizan en su importancia para el ejercicio profesional. El cuarto hace alusión a estudios sobre el impacto que tiene la formación en ética para el proceso de toma decisiones éticas. En el quinto se ubican aquellos artículos que relacionan la formación en ética con la cultura de las organizaciones y finalmente se clasifican los artículos que discuten sobre el papel del género en los aprendizajes sobre ética en las organizaciones.

El análisis de la información permite identificar tendencias y futuras líneas de investigación. Por ejemplo se aprecia que los debates se han alejado de sí es pertinente enseñar ética o no y se han centrado en buscar las mejores pedagogías para que la formación sea efectiva (McWilliams y Nahavand, 2006). Es por ello que la metodología de enseñanza juega un papel protagónico en las referencias consultadas (53,92\%) pues genera amplia producción académica en la actualidad. Igualmente ejes temáticos como la enseñanza de la ética en las universidades $(26,47 \%)$ o la importancia de la formación en ética para los profesionales $(7,84 \%)$ se relacionan y complementan. Estos tres componentes se agrupan en la tendencia más robusta denominada aspectos pedagógicos para la formación en ética en las organizaciones (90 artículos de los 102 consultados - 88,24\%). También se evidencian otras líneas temáticas que relacionan la formación en ética con temas organizacionales como toma de decisiones éticas $(3,92 \%)$ y cultura organizacional ética $(2,94 \%)$, 
y aunque tienen menos producción bibliográfica $(6,86 \%)$, emergen para aportar en la consolidación de la comunidad científica. De manera adicional se incluye la tendencia del género como un tema emergente en la discusión sobre formación en ética en las organizaciones (2,94\%). A continuación se hace una discusión de cada una de estas tendencias, resaltando las ideas y autores destacados respecto al tema.

Tabla 4: Temas que se abordan en los artículos consultados

\begin{tabular}{clcc}
\hline$N^{\circ}$ & Tema del estudio & No Artículos & Porcentaje \\
\hline 1 & Aspectos pedagógicos para enseñar ética en las organizaciones & 55 & $53,92 \%$ \\
\hline 2 & La enseñanza de la ética organizacional en las universidades & 27 & $26,47 \%$ \\
\hline 3 & Importancia de la formación en ética organizacional para los profesionales & 8 & $7,85 \%$ \\
\hline 4 & Formación para la toma de decisiones éticas & 4 & $3,92 \%$ \\
\hline 5 & Formación en ética y cultura organizacional ética & 3 & $2,94 \%$ \\
\hline 6 & Formación en ética y género & 3 & $2,94 \%$ \\
\hline 7 & Otros temas relacionados & 2 & $1,96 \%$ \\
\hline & Total & 102 & $100 \%$ \\
\hline
\end{tabular}

\section{Aspectos pedagógicos para la formación en ética organizacional}

Uno de los temas que ha guiado la discusión en formación en ética en las organizaciones ha sido el impacto que tiene su enseñanza, es decir, responder si es posible enseñar ética a estudiantes o miembros de una organización, de tal manera que modifiquen positivamente sus comportamientos (Sims y Felton, 2006). Aunque en la comunidad científica hay disenso sobre la utilidad de la enseñanza de la ética en adultos, varios estudios han mostrado que la formación en ética puede mejorar la sensibilidad, el razonamiento y el juicio respecto a dilemas éticos del ámbito laboral, logrando avances en la toma de decisiones fundamentada en principios éticos (Weber, 2015, 1990; Arce y Gentile, 2015; McWilliams y Nahavand, 2006; Ritter, 2006; Adkins y Radtke, 2004). Se asume entonces que enseñar ética es útil y que la formación universitaria debe comprometerse de manera categórica con el desarrollo moral de los estudiantes, pues las competencias profesionales y la ética deben estar en sinergia constante, aunque no hay lineamientos claros sobre las estrategias metodológicas que se deben implementar (Osuna y Luna, 2011).

El creciente acuerdo sobre la importancia de la enseñanza de la ética organizacional ha generado que las investigaciones actuales se inclinen hacia el cuestionamiento sobre su efectividad y metodología de enseñanza. Por ejemplo se busca dar respuesta a preguntas como ¿de qué manera es posible medir el impacto de un programa de formación en ética en las organizaciones?, ¿cómo se debe llevar a cabo un programa de formación en ética para que los profesionales logren una aprehensión del actuar ético en el ámbito laboral? o ¿cuál debe ser el objetivo de un curso en ética organizacional? (Baden, 2014; Sims y Felton, 2006; Ritter, 2006). Las respuestas a estos cuestionamientos han motivado el desarrollo de un gran número de investigaciones teóricas y empíricas que aportan a la consolidación del campo de estudio a través de la formulación de estrategias y metodologías para la formación en ética tanto de estudiantes universitarios de pregrado y posgrado, como de colaboradores y profesionales que ejercen sus disciplinas.

Algunas investigaciones que retoman el aspecto metodológico como factor determinante para que la enseñanza de la ética organizacional sea un proceso efectivo son: Rodríguez et al., 2016; Cheng, 2015; Fischbach, 2015; Maclagan, 2015; Shapira-Lishchinsky, 2015; Gu y Neesham, 2014; Warren et al., 2014; Slocum et al., 2014; Singer, 2013; Osuna y Luna, 2011; Mayhew y Murphy, 2009; Falkenberg y Woiceshyn, 2008; Gibson, 2008; McWilliams y Nahavand, 2006; Sims, 2002. De ellas, se destacan asuntos tales como: rol del docente, importancia del contexto, pertinencia de los contenidos, enfoque pedagógico y estrategias de enseñanza como el estudio de caso, el aprendizaje experiencial y la discusión reflexiva-conceptual.

En la literatura se destaca el estudio de caso como una herramienta adecuada, ya que permite la reflexión personal y la clarificación del marco conceptual de un individuo para decidir qué es y qué no es un comportamiento ético. Además, promueve el pensamiento crítico y la discusión de los problemas que surgen en el aula y en el lugar de trabajo. Esta estrategia de aprendizaje es adecuada cuando utiliza casos reales y actuales. A los participantes se les proveen situaciones críticas de la cotidianidad en la que deben identificar los elementos morales que se están asumiendo o vulnerando. Con ello los estudiantes tienen herramientas analíticas para comprender los contextos que pueden vivenciarse en diversos planos de las organizaciones. Los casos promueven la reflexión personal y reconocen la importancia de la aplicación de los valores morales en la vida personal, profesional y en la resolución de problemas con los otros (Cheng, 2015; Fischbach, 2015; Maclagan, 2015; Singer, 2013; Falkenberg y Woiceshyn, 2008; Gibson, 2008; McWilliams y Nahavand, 2006).

Para Rodríguez et al. (2016) y Shapira-Lishchinsky (2015) el enfoque constructivista de la enseñanza facilita el desarrollo de competencias para el comportamiento moral. De acuerdo con la teoría constructivista, el 
aprendizaje es un proceso activo en el que los participantes construyen nuevos conocimientos sobre la base de sus saberes y experiencias actuales y pasadas. En este sentido, el aprendizaje experiencial se presenta como una estrategia eficaz, ya que las personas tienen aprendizajes más significativos cuando entran en contacto con sus propias vivencias y reflexionan sobre lo cotidiano (Rodríguez et al., 2014). Se recomienda este tipo de estrategia, debido a que los participantes asumen una percepción más real de las situaciones y reconocen sus fortalezas y debilidades. Además porque se puede articular con otro tipo de actividades como el estudio de caso o la discusión reflexiva-conceptual.

Mayhew y Murphy (2009) a través de un estudio comparativo para analizar cómo los sujetos asumen valores éticos a través de preguntas sobre situaciones de la cotidianidad, concluyeron que la educación en ética organizacional tiene efectos positivos en quienes la reciben, pero tal aprendizaje tiene un mayor impacto si va acompañado de otras estrategias, como por ejemplo la presión social a través de la divulgación pública. En esta misma línea Gu y Neesham (2014) sugieren utilizar un enfoque basado en la identidad que incluye métodos de autorreflexión y estrategias extraídas de la psicología social.

Respecto al papel que desempeña el docente en la formación en ética, se destaca su rol como orientador y guía del aprendizaje, ya que su responsabilidad tendrá un impacto en la creación de espacios seguros y amigables para las reflexiones analíticas, donde los participantes sientan la confianza para compartir ideas y sentimientos. El docente debe posibilitar la discusión, pues las puestas comunes son esenciales para realizar y tomar las indicaciones que garantizarán el intercambio colaborativo, pero también debe propiciar espacios individuales que permitan la formación en valores morales y preparar a los participantes en los tipos de dilemas éticos que se encontrarán en sus áreas laborales. Las precisiones conceptuales son fundamentales para que no se asuman definiciones de manera superficial y que puedan vislumbrar cualquier situación sobre el plano de la ética (Cheng, 2015; Slocum et al., 2014; Felton y Sims, 2005). Como se trata de formación en ética para adultos el docente es quien debe hacer el esfuerzo para adecuar su programa a las necesidades de los participantes, su rol es ser facilitador del aprendizaje a través de metodologías flexibles (Castaño y Garín, 2012).

En síntesis, los aspectos metodológicos para llevar a cabo un proceso de formación en ética organizacional que sea significativo es una discusión vigente en la literatura. Entre las conclusiones sobre esta tendencia se destaca que su implementación requiere del diseño de un programa de formación con una metodología estructurada que tenga en cuenta: la didáctica más pertinente para el aprendizaje de adultos, el modelo pedagógico, las mediaciones e instrumentos de aprendizaje, el rol que asumen los docentes y estudiantes, el contexto y la forma de evaluar el programa (una medición de impacto y no sólo de cumplimiento). A continuación se relacionan los aspectos consolidados por McWilliams y Nahavandi (2006) quienes recogen lo planteado por diversos autores y brindan unas pautas metodológicas que se consideran oportunas para mejorar el proceso de enseñanza-aprendizaje de la ética en las organizaciones: (i) Iniciar por la exposición y clarificación de conceptos filosóficos sobre la ética; (ii) La teoría debe complementarse con la aplicación de los conceptos a situaciones laborales cotidianas; (iii) Los temas deben ser importantes para los participantes, de tal manera que se genere una conexión emocional entre sus valores y opiniones personales con los dilemas abordados; (iv) Se deben relacionar los conceptos con temas de interés, esto ayuda a que haya retención a largo plazo; y (v) Debe haber un ambiente favorable para la expresión de opiniones, la argumentación y el debate.

Con base a las referencias consultadas es posible afirmar que los autores que están investigando en los aspectos pedagógicos de la formación en ética organizacional, consideran que este proceso tiene efectos positivos para las personas y las organizaciones involucradas, pero que sin duda la metodología bajo la cual se lleve a cabo influye en que este saber tenga un mayor impacto. Estudios teóricos y empíricos se están desplegando en la comunidad académica para validar las hipótesis existentes, con el convencimiento de que aunque no haya certeza de la reacción que los estudiantes tengan ante los desafíos éticos en el mundo real, no se debe omitir la enseñanza de la ética (Wang y Calvano, 2015).

\section{Formación en ética en las organizaciones y género}

Algunos artículos sugieren que hay factores que influyen en la interiorización de la formación en ética en las organizaciones. El contexto, la cultura, la edad y el género son algunos de ellos. Este último es uno de los elementos que han sido debatidos y es recurrente en la presente revisión (Jonson et al., 2016, 2015; Wang y Calvano, 2015; Wurthmann, 2013; Ritter, 2006; Gilligan, 1985). Por ejemplo, Wang y Calvano (2015), en una investigación con estudiantes del área de administración, plantean la relación entre género y juicio moral, el impacto de la formación en ética organizacional y las relaciones entre las características personales y la moralidad. Entre los resultados se destaca que las mujeres generalmente son más propensas a actuar de forma ética que los hombres, pues mientras que los hombres se inclinan a tomar decisiones basadas en nociones de justicia, las mujeres se inclinan por tener en cuenta los contextos 
situacionales y los constructos mediados por el simbolismo social y cultural. En contraposición, los hombres son más sensibles a la formación en ética que las mujeres, pues después de participar en un proceso de formación tienden en mayor medida a responder éticamente en situaciones de negocios.

Esta conclusión se fundamenta en estudios desarrollados por Gilligan (1985) quien hace parte de una corriente de la filosofía moral denominada ética del cuidado, la cual considera que el hombre y la mujer actúan de manera diferente ante algunas situaciones. Señala que por su carácter sensible las mujeres privilegian acciones morales enmarcadas en valores como el cuidado, el amor, la protección, la solidaridad y la responsabilidad. A diferencia de los hombres quienes, generalmente, dan relevancia a la legalidad y la justicia. Sobre esto, Ritter (2006) plantea que es probable que, aunque los hombres son conscientes de los principios éticos, se identifican a un grado más fuerte con el paradigma de negocio tradicional que sugiere la prioridad de las utilidades sobre las consideraciones éticas. Mientras que en amplios casos las mujeres han incluido nuevas formas de ver los negocios y están dispuestas a hacer concesiones a los demás.

Así pues, de las investigaciones analizadas sobre esta tendencia, se puede inferir que un proceso de formación en ética incide de alguna manera en el actuar de quienes participan en el mismo, sean hombres o mujeres. El género es un elemento que puede influir para definir que un proceso de formación es efectivo. Sin embargo, no es posible generalizar que sea un factor significativo en la explicación de las diferencias y por el contrario estudios como el de Jonson et al. (2015) afirman que entre hombres y mujeres no hay diferencias significativas con respecto a la formación en ética. En este sentido, se requiere de la implementación de investigaciones que aporten evidencia teórica y empírica suficiente para aclarar la relación entre el género y la formación en ética organizacional.

\section{Formación en ética en las organizaciones y toma de decisiones éticas}

En esta tendencia, las referencias consultadas muestran un acuerdo entre diversos autores sobre que el proceso formativo influencia de manera positiva la toma de decisiones éticas y el juicio moral de las personas. Dicho autores afirman que los participantes en los procesos formativos adquieren elementos para reconocer a la luz del raciocinio ético las opciones más adecuadas (Verma et al., 2016; Neesham y Gu, 2015; Shapira-Lishchinsky, 2015; May et al., 2014; Gu y Neesham, 2014; Ritter, 2006; Jones, 1991; Treviño, 1986; Colby et al., 1980; Kohlberg, 1976).

La teoría del desarrollo moral cognitivo de Kohlberg (1976) se pone de manifiesto como una herramienta para determinar lo que es correcto o incorrecto, haciendo énfasis en los aspectos cognitivos y en los aspectos de razonamiento en la toma de decisiones morales. También aborda cómo los procesos cognitivos de la moral se vuelven más complejos y sofisticados con el desarrollo. Su tesis principal expone que existen seis estadios de desarrollo moral que evolucionan de acuerdo con las reflexiones morales del bien y el mal. Para Kohlberg, la capacidad del juicio moral se desarrolla en tres etapas: una preconvencional, donde los sujetos aún no han adquirido las habilidades o elementos simbólicos respecto a las convenciones morales socialmente construidas; una convencional, en las que se adquieren los elementos morales, y una posconvencional, donde los individuos asumen posturas críticas y analíticas sobre la moralidad.

El enfoque educativo de Kohlberg propone desarrollar el juicio moral a través del intercambio de ideas respetando las fronteras de la libertad y de discusiones sobre dilemas morales que son entendidos como problemas en los que se produce un significativo intercambio de valores, debido a que se pueden analizar las contradicciones que manen de los argumentos expuestos. Para el autor, el juicio moral se desarrolla en cada individuo a través de fases que llevan explícitamente un aprendizaje irreversible, en las que se adquieren nuevos niveles de conocimiento, de valoración y de acción. Desde esta postura el ser humano decide en forma libre y racional lo que le corresponde hacer desde un punto de vista moral, rescatando las cualidades de sujeto autónomo. Aunque no exentos de críticas, los planteamientos de Kohlberg representan una contribución a la investigación en formación en ética organizacional, ya que asumen que la moral puede y debe ser enseñada, da pautas para abordar el tema desde lo metodológico y proporciona una definición del constructo juicio moral, lo cual fundamenta la toma de decisiones de cada sujeto (Colby et al., 1980).

En la toma de decisiones son constantes los dilemas éticos, donde la indagación por lo que es correcto o incorrecto es un asunto complejo y en donde es necesario identificar variables que permitan comprender las situaciones y la actuación hacia las mismas. Por ejemplo, en un contexto laboral, los valores tienen incidencia en el proceder de los individuos en sus puestos de trabajo, lo que sugiere que la dimensión de lo ético está relacionada con la formación de las personas, de sus contextos y de su visión del mundo. Por ello, ante problemas, los individuos reaccionan y asumen posturas específicas dependiendo de su etapa de desarrollo moral cognitivo (Treviño, 1986). 
En las organizaciones las personas exploran situaciones éticas cuando deben verse cara a cara con sus propios valores y con su habilidad de gestionar y de resolver las situaciones que ameritan una intervención en el área laboral. Mejorar el proceso de toma de decisiones es esencial para el desarrollo de la organización, ya que afecta a los gestores y líderes de las organizaciones, influye en los campos de producción en los cuales se enfocan las compañías e incide en sus grupos de interés (Treviño, 1986). En este sentido, con la formación en ética se busca capacitar a los empleados para que sean más conscientes de los problemas éticos con que se enfrentan en el trabajo y para que incrementen las habilidades para la toma de decisiones éticas (Weber, 2015).

Felton y Sims (2005) argumentan que quienes reciban formación en ética deben estar altamente interesados en conocer las visiones de los otros, deben aprender a defender, articular y a persuadir a sus pares con sus visiones de la realidad en búsqueda del bien colectivo. Por ello el proceso de enseñanza implica que los debates en torno a las problemáticas sean de carácter vigoroso y que generen un desafío para la toma de decisiones de los participantes, puesto que es allí donde se encontrará lo significativo del aprendizaje que se desea alcanzar.

En general de la revisión realizada sobre esta tendencia, es posible concluir que hay un acuerdo respecto a que la formación en ética es un medio importante para fomentar la toma de decisiones éticas en las organizaciones. La efectividad de este proceso depende en gran medida de los esfuerzos que despliegue la organización para llevarla a cabo, pues influyen tanto las ideologías individuales, como los valores éticos de la organización (Verma et al., 2016). Así las cosas, se aprecia que explorar tal relación puede ser una línea de investigación prometedora para fomentar los estudios en formación en ética en las organizaciones, pues es un tema vigente y que no ha sido estudiado con suficiencia.

\section{Formación en ética y cultura organizacional ética}

La cultura organizacional es entendida como un sistema particular de símbolos influidos por la historia y la sociedad en que se desenvuelve la organización y que van generando su identidad, es flexible y se transforma de acuerdo con los factores de contingencia generados por los empleados y el contexto (Allaire y Firsirotu, 1992). Méndez (2000) la define como la conciencia colectiva que se expresa en el sistema de significados compartidos por los integrantes de la organización, que los identifica y diferencia de otros, institucionalizando y estandarizando sus conductas sociales. Así mismo, la cultura organizacional influye en el comportamiento y en el desempeño de los empleados, pues a través de esta es posible establecer parámetros morales con los cuales los individuos pueden vincularse o identificarse (Treviño y Nelson, 2010; Valentine y Fleischman, 2004). Cuando una organización le apunta a la inclusión de parámetros morales como eje de su cultura organizacional, se le denomina cultura organizacional ética. En este sentido, una organización puede contribuir al desarrollo moral de un individuo, al alentar una cultura que permita a sus integrantes la toma de decisiones éticas y el cumplimiento de responsabilidades individuales y sociales.

Autores como Rodríguez (2015), Warren (2014), Treviño y Nelson (2010) y Valentine y Fleischman (2004) asumen que la formación en ética puede generar una dinámica positiva en la cultura de las organizaciones. De esta manera se fortalece una cultura organizacional ética, entendida como aquella que "formal e informalmente, promueve la toma de decisiones éticas y el comportamiento ético. Formalmente, a través de la ideología, de la declaración de valores y principios organizacionales, de un programa de cumplimiento legal, de un código de ética y de la instauración de una línea para whistleblowers. Informalmente, a través del ejemplo de los líderes de la organización, de los mitos que refuerzan el mensaje ético y de la práctica de los valores declarados" (Rodríguez, 2015, p. 128).

Sin embargo, para que la formación en ética potencie una cultura organizacional ética, se requiere que el proceso esté vinculado a un programa de mayor amplitud en el cual se establezcan diversas estrategias en las que los valores sean el marco de referencia para la toma de decisiones y para el comportamiento de los empleados, y donde la cultura ética se irradie interna y externamente (Kaptein, 2015). Para Rodríguez los programas de ética se deben "basar en la integridad y en la construcción de confianza entre los grupos de interés, buscando hacer que la ética sea parte central de la cultura organizacional, lo cual traerá como resultados el incremento de la eficiencia en las operaciones, mayor compromiso de los empleados, mayor lealtad de los inversionistas, mayor satisfacción de los clientes, mejor desempeño financiero y mejores relaciones con los grupos de interés" (2015, p. 127).

También se destaca que las organizaciones que quieran fortalecer su dimensión ética a través de la formación, deben tener el apoyo de su gobierno (socios, accionistas, junta directiva y gerentes de todos los niveles), para que se garanticen los recursos económicos, logísticos y de talento humano para llevarlo a cabo. Además, para lograr estos cambios, la organización y sus integrantes requieren de apertura hacia el aprendizaje, en este caso desde lo ético. Para Marulanda et al. (2016) la adquisición de competencias para el aprendizaje organizacional está influida por la cultura de la organización, pues es la cultura quien 
establece los parámetros sobre el conocimiento que debe ser adquirido. De igual forma hay que tener en cuenta que el aprendizaje organizacional es un tema que debe ser entendido en "términos de obtención y asimilación de conocimientos, que modifican el comportamiento y la estructura cognitiva de la organización y permiten generar o reconfigurar capacidades y competencias para mejorar o mantener su desempeño y resultados" (Cardona y Calderón, 2006, p. 16).

Respecto a las ventajas que se pueden derivar del proceso formativo y su relación con la cultura organizacional ética, Valentine y Fleischman (2004) señalan que en las organizaciones que tienen formalizados estos programas de formación, los empleados tienen una percepción más positiva de sus lugares de trabajo. En esta misma línea, Warren et al. (2014) resaltan los siguientes beneficios: i) los integrantes de la organización diferencian entre comportamientos éticos y no éticos, lo que conlleva a respuestas apropiadas; ii) los empleados perciben el comportamiento ético como parte integral de sus roles organizacionales; iii) puede mejorar la percepción de los trabajadores respecto a la eficacia de la organización y iv) se refuerza la estructura normativa que influye en la cultura organizacional.

Sobre esta tendencia es posible concluir que la relación entre formación en ética y cultura organizacional ética debe ser explorada con mayor profundidad. Se requieren de estudios que refuten o complementen los hallazgos existentes, los cuales, hasta el momento, reflejan que la formación para el desarrollo de las sensibilidades éticas es un proceso pertinente para las organizaciones. Esto debido a que sus integrantes están expuestos a dilemas éticos, por lo que deben comprender y afianzar sus propios valores y alinearlos con los de la organización.

En general, se considera que la formación en ética es un pilar para el liderazgo que permite la proyección de las organizaciones, de sus metas, de los sujetos que la integran y de la responsabilidad social que las convoca. En esta medida resulta fundamental que exista un modelo idóneo de enseñanza de la ética que se implemente en las organizaciones, pero también en los diversos niveles educativos, de tal manera que un futuro los habitantes organizacionales tengan un razonamiento ético fortalecido.

\section{CONCLUSIONES}

De la literatura presentada, su análisis y discusión se puede concluir lo siguiente:

(i) Se destacan cuatro tendencias de investigación: aspectos pedagógicos y didácticos que se deben tener en cuenta para que un programa de formación en ética organizacional sea efectivo; incidencia del género en la efectividad de la formación en ética organizacional; la formación en ética como potenciadora de habilidades para la toma de decisiones éticas de los empleados de una organización y la relación entre formación en ética y fortalecimiento de una cultura organizacional ética;

(ii) Los estudios que se centran en los aspectos pedagógicos para que la formación en ética sea efectiva son los más frecuentes $(88,23 \%$ de las referencias consultadas). Son investigaciones, generalmente empíricas y cuantitativas, que hacen diversas propuestas metodológicas incluyendo aspectos como objetivos de aprendizaje, contenidos, espacios, rol del docente, rol de los estudiantes, didáctica y evaluación;

(iii) e identifican como líneas de investigación promisorias la exploración de las relaciones entre formación en ética y toma de decisiones éticas; formación en ética y variables como el género y formación en ética y cultura organizacional ética, pues a través de la revisión realizada fueron tendencias referenciadas con un bajo porcentaje: 3,92\%, 2,94\% y 2,94\% respectivamente. Además serían campos de estudio que pueden sentar bases teóricas y empíricas que contribuyan al fortalecimiento del tema en una comunidad científica en crecimiento y que referencia la formación en ética como una línea emergente dentro del campo de estudio de la ética en las organizaciones;

(iv) Uno de los desafíos del área es que para dar peso científico a los desarrollos teóricos, se requiere de la implementación de investigaciones empíricas con parámetros similares de medición, pero en diferentes escenarios, de tal manera que se contraste y valide la teoría existente y se puedan ir fortaleciendo acuerdos sobre el tema;

(v) Finalmente, es posible concluir que las revisiones sistemáticas de literatura son una estrategia pertinente para encontrar material bibliográfico relevante, información que posteriormente puede ser actualizada con los avances que se vayan generando. La plataforma web Tree of Science se presenta como una herramienta de investigación útil y efectiva para aquellas investigaciones que requieren de la realización de una búsqueda sistemática. 


\section{REFERENCIAS}

AACSB, International: Business Accreditation Standards 2013 (en línea), http://www.aacsb.edu/accreditation/standards. Acceso: 11 de noviembre (2016)

Adkins, N. y R. Radtke, Students' and faculty members' perceptions of the importance of business ethics and accounting ethics education: Is there an expectations gap, Journal of Business Ethics, 51(3), 279-300 (2004)

Allaire, Y. y M. Firsirotu, Teorías sobre la cultura organizacional, en Cultura organizacional por H. Abravanel, Y. Allaire, M. Firsirotu, B. Hobbs, R. Poupart y J.J. Simard (Eds.), pp 3-37, LEGIS, Bogotá, Colombia (1992)

Arce, D.G. y M.C. Gentile, Giving Voice to Values as a Leverage Point in Business Ethics Education, Journal of Business Ethics, 131(3), 535-542 (2015)

Baden, D., Look on the Bright Side: A Comparison of Positive and Negative Role Models in Business Ethics Education, The Academy of Management Learning \& Education, 13(2), 154-170 (2014)

Buckley, R. y J. Caple, La formación: Teoría y práctica, Ediciones Díaz de Santos, Madrid, España (1991)

Bunge, M., La ciencia, su método y su filosofía, Editorial Siglo XX, Buenos Aires, Argentina (1978)

Calderón, G., Aprender a investigar investigando, Editorial UN, Manizales, Colombia (2005)

Cameron, R. y C. O'Leary, Improving Ethical Attitudes or Simply Teaching Ethical Codes? The Reality of Accounting Ethics Education, Accounting Education an International Journal, 24(4), 275290 (2015)

Cardona J. A. y G. Calderón, El Impacto del Aprendizaje en el Rendimiento de las Organizaciones, Cuadernos de administración, 19(32), 11-43 (2006)

Castaño, E. y A. Garín, Incorporación de Personas Adultas a los Estudios Universitarios: Aprendizaje Permanente para Arquitectos Técnicos, Formación Universitaria, 5(3), 17-26 (2012)

Chan, K.C., A. Fung, H.G. Fung y J. Yau, A Citation Analysis of Business Ethics Research: A Global Perspective, Journal of Business Ethics, 136(3), 557-573 (2016)

Cheng, P., University Lecturers' Intention to Teach an Ethics Course: A Test of Competing Models, Journal of Business Ethics, 126(2), 247-258 (2015)

Colby, A., J. Gibbs, L. Kohlberg, B. Speicher y D. Candee, The Measurement of Moral Judgment (Vol. I), Center for Moral Education Harvard University, Cambridge, England (1980)

De la Torre, I., La Formación y las Organizaciones: Los Acuerdos Nacionales de Formación Continua, Revista Española de Investigaciones Sociológicas, 77, 15-34 (1997)

Falkenberg, L. y J. Woiceshyn, Enhancing Business Ethics: Using Cases to Teach Moral Reasoning, Journal of Business Ethics, 7(3), 213-217 (2008)

Felton, E. y R. Sims, Teaching Business Ethics: Targeted Outputs, Journal of Business Ethics, 60(4), 377391 (2005)

Ferreira, I., G.U. González y P. Coello, Systematic Reviews and Meta-Analysis: Scientific Rationale and Interpretation, Revista Española de Cardiología, 64(8), 688-696 (2011)

Fischbach, S., Ethical Efficacy as a Measure of Training Effectiveness: An Application of the Graphic Novel Case Method versus Traditional Written Case Study, Journal of Business Ethics, 128(3), 603-615 (2015)

Gibson, P., Teaching Ethical Decision Making: Designing a Personal Value Portrait to Ignite Creativity and Promote Personal Engagement in Case Method Analysis, Ethics \& Behavior, 18(4), 340-352 (2008)

Gilligan, C., La moral y la teoría. Psicología del desarrollo femenino, Fondo de Cultura Económica, México D.F., México (1985) 
Gu, J. y C. Neesham, Moral Identity as Leverage Point in Teaching Business Ethics, Journal of Business Ethics, 124(3), 527-536 (2014)

Guillén, M., Ética en las organizaciones. Construyendo confianza, Pearson, Madrid, España (2006)

Guisán, E., Razón y pasión en ética. Los dilemas de la ética contemporánea, Editorial Anthropos, Barcelona, España (1990)

Hare, R., El lenguaje de la moral, Universidad Nacional Autónoma de México, Instituto de Investigaciones Filosóficas, México D.F., México (1975)

Hernández, R., C. Fernández y P. Baptista, Metodología de la investigación, 5ae edición, McGrawHill, México D.F., México (2010)

Hummel, K., D. Pfaff y K. Rost, Does Economics and Business Education Wash Away Moral Judgment Competence? doi:10.1007/s10551-016-3142-6 (en línea), Journal of Business Ethics, 1-19 (2016)

Jones, T., Ethical Decision Making by Individuals in Organizations: An Issue-Contingent Model, The Academy of Management Review, 16(2), 366-395 (1991)

Jonson, E.P., L. McGuire y B. Cooper, Does Teaching Ethics Do Any Good? Education and Training, 58 (4), 439-454 (2016)

Jonson, E.P., L. McGuire y D. O'Neill, Teaching Ethics to Undergraduate Business Students in Australia: Comparison of Integrated and Stand-Alone Approaches, Journal of Business Ethics, 132(2), 477-491 (2015)

Kaptein, M., The Effectiveness of Ethics Programs: The Role of Scope, Composition, and Sequence, Journal of Business Ethics, 132(2), 415-431 (2015)

Kohlberg, L., Moral stages and moralization. The cognitive-developmental approach, en Moral Development and Behavior por T. Likona, pp. 170-205, Holt, Rinehart \& Winston, Nueva York, USA (1976)

Maclagan, P., Moral Dilemmas, Moral Reasons and Moral Learning: Interpreting a Real Case in Terms of Particularistic Theory, Business Ethics: A European Review, 24(3), 221-236 (2015)

Marulanda, C., M. López y F. López, La Cultura Organizacional y las Competencias para la Gestión del Conocimiento en las Pequeñas y Medianas Empresas (PYMEs) de Colombia, Información Tecnológica, 27(6), 3-10 (2016)

Mathews, C. y S. Gandel. The 5 Biggest Corporate Scandals of 2015, Fortune (en la web: http://fortune.com/2015/12/27/biggest-corporate-scandals-2015/. Acceso: 27 de diciembre (2015)

May, D., M. Luth y C. Schwoerer, The Influence of Business Ethics Education on Moral Efficacy, Moral Meaningfulness, and Moral Courage: A Quasi-Experimental Study, Journal of Business Ethics, 124(1), 67$80(2014)$

Mayhew, B. y P. Murphy, The Impact of Ethics Education on Reporting Behavior, Journal of Business Ethics, 86(3), 397-416 (2009)

McWilliams, V. y A. Nahavandi, Using Live Cases to Teach Ethics, Journal of Business Ethics, 67(4), 421$433(2006)$

Méndez, C. E., Un marco teórico para el concepto de cultura corporativa, Universidad del Rosario, Bogotá, Colombia (2000)

Neesham, C. y J. Gu, Strengthening Moral Judgment: A Moral Identity-Based Leverage Strategy in Business Ethics Education, Journal of Busindess Ethics, 131(3), 527-534 (2015)

Osuna, C. y E. Luna, Valores Éticos en la Formación Universitaria de las Áreas de Ciencias Naturales e Ingeniería y Tecnología, en el Contexto de la Sociedad del Conocimiento, Formación Universitaria, 4(5), 29$36(2011)$

Ritter, B., Can Business Ethics be Trained? A Study of the Ethical Decision-Making Process in Business Students, Journal of Business Ethics, 68(2), 153-164 (2006) 
Robledo, S., G.A. Osorio y C. López, Networking en Pequeña Empresa: Una Revisión Bibliográfica Utilizando la Teoría de Grafos, Revista Vínculos, 11(2), 6-16, (2014)

Rodríguez, M.P., M.A. Pantoja y F. Cantor, Uso Ético del Poder Gerencial: Propuesta de un Programa para la Formación de Estudiantes de Ingeniería, Formación Universitaria, 9(3), 87-98 (2016)

Rodríguez, M.P., Culturas Organizacionales Éticas, Virajes: Revista de Antropología y Sociología, 17(1), 107-134 (2015)

Rodríguez, M.P., F. Cantor, M.A. Pantoja, J. Toro y A.F. Vásquez, Formación Gerencial en Uso de Poder: Un Enfoque Ético, Editorial UN, Bogotá, Colombia (2014)

Rodríguez, M.P., C.F. Castaño, V. Osorio, H.F. Zuluaga y V. Duque. La auditoría ética: herramienta para fortalecer la integridad del carácter organizacional, Innovar, 16(27), 26-46 (2006)

Shapira-Lishchinsky, O., Simulation-Based Constructivist Approach for Education Leaders, Educational Management Administration \& Leadership, 43(6), 972-988 (2015)

Sims R., Debriefing Experiential Learning Exercises in Ethics Education, Teaching Business Ethics, 6(2), 179-197 (2002)

Sims, R. y E. Felton, Designing and Delivering Business Ethics Teaching and Learning, Journal of Business Ethics, 63(3), 297-312 (2006)

Singer, A., Teaching Ethics Cases: A Pragmatic Approach, Business Ethics: A European Review, 22(1), 16$31(2013)$

Slocum, A., S. Rohlfer y C. González-Canton, Teaching Business Ethics Through Strategically Integrated Micro-Insertions, Journal of Business Ethics, 125(1), 45-58 (2014)

Soltani, B, The Anatomy of Corporate Fraud: A Comparative Analysis of High Profile American and European Corporate Scandals, Journal of Business Ethics, 120(2), 251-274 (2014)

Treviño, L.K. y Nelson, K.A., Managing business ethics, $5^{\text {th }}$ edition, Wiley, New Jersey, USA (2010)

Treviño, L.K., Ethical Decision Making in Organizations: A Person-Situation Interactionist Model, The Academy of Management Review, 11(3), 601-617 (1986)

Valentine, S. y G. Fleischman, Ethics Training and Businesspersons. Perceptions of Organizational Ethics, Journal of Business Ethics, 52(4), 391-400 (2004)

Velásquez, J.D., Una Guía Corta para Escribir Revisiones Sistemáticas de Literatura, DYNA, 81(187), 9-10 (2014)

Verma, P., S. Mohapatra y J. Löwstedt, Ethics Training in the Indian IT Sector: Formal, Informal or Both? Journal of Business Ethics, 133 (1), 73-93 (2016)

Wang, I. y L, Calvano, Is Business Ethics Education Effective? An Analysis of Gender, Personal Ethical Perspectives, and Moral Judgment, Journal of Business Ethics, 126(4), 591-602 (2015)

Weber, J., Investigating and Assessing the Quality of Employee Ethics Training Programs Among US Based Global Organizations, Journal of Business Ethics, 129(1), 27-42 (2015)

Weber, J., Measuring the Impact of Teaching Ethics to Future Managers: A Review, Assessment, and Recommendations, Journal of Business Ethics, 9(3), 183-190 (1990)

Warren, D.E., J.P. Gaspar y W.S. Laufer, Is Formal Ethics Training Merely Cosmetic? A Study on Ethics Training and Ethical Organizational Culture, Business Ethics Quarterly, 24(1), 85-117 (2014)

Wurthmann, K., A Social Cognitive Perspective on the Relationships between Ethics Education, Moral Attentiveness, and PRESOR, Journal of Business Ethics, 114(1), 131-153 (2013) 
